\title{
A Statistical Investigation of Prominence Fine Structure in the EUV
}

\author{
S. Pojoga ${ }^{1}$, A. G. Nikoghossian ${ }^{2}$ and Z. Mouradian \\ Observatoire de Paris-Meudon, 92195 Meudon Cedex, France
}

\begin{abstract}
A statistical approach is proposed for the study of the prominence fine structure. Intensity variations in several EUV lines are measured in different regions of prominences. The expected number of fine structural elements is derived.
\end{abstract}

\section{Introduction}

An important problem of solar prominences is to determine the geometrical and physical characteristics of fine structure elements which are difficult to detect directly from observations. Several methods have been used to derive the number of the sub-resolution fine structures (Orrall and Schmahl 1980, Engvold et al. 1989, Zirker and Koutchmy 1990). The present note suggests a new approach based on spatial fluctuations of the brightness of prominences.

\section{Statement of the Problem}

We propose to study the prominence spectrum by considering the transfer of radiation through randomly distributed fine structures. We compare the theoretical and observational intensity distributions and their characteristics, thus providing further information to estimate the geometrical and physical parameters of prominences. The statistical parameters measured and computed are the mean intensity, $\langle I\rangle$ and the relative mean square deviation (RelMSD), $\delta=\left\langle I^{2}\right\rangle /\langle I\rangle^{2}-1$. The EUV multispectral observations allow us to consider several layers of the prominence-corona atmosphere, from the cool prominence to the hot coronal plasma.

The main hypothesis is that the observed intensities and their fluctuations are due only to the random number of structures on the line of sight, which is distributed according to the Poisson law. An idealized model problem accounting for the random character of the initial release of radiation energy was treated by Nikoghossian et al. (1997). We also suppose that the structural elements are identical and have a slab-like geometry such that each structure contains a central cool slab separated from the hot corona by two transition

\footnotetext{
${ }^{1}$ Astronomical Institute of Romanian Academy, 75212 Bucarest, Romania

${ }^{2}$ Byurakan Astrophysical Observatory, 378433 Byurakan, Armenia
} 

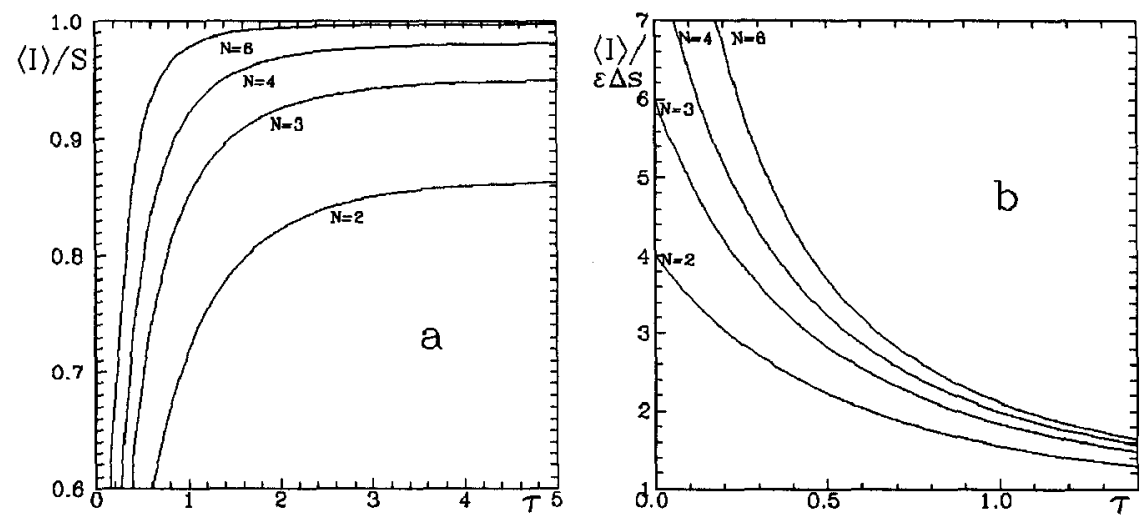

Figure 1. Theoretical mean intensity $\langle I(N, \tau)\rangle$ : a) Core emission, b) Interface emission.

regions. A detailed treatment of the problem, including a thread-like cylindrical model, is studied by Pojoga et al. (1998). Depending on the region of spectral line formation, we consider two approaches for the radiative transfer: a) Core emission. The line forms only in the central layer of the structure, which can be more or less optically thick. We neglect absorption through the interface and corona. b) Interface emission. The region of the line formation is the transition zone of the features. This is considered optically thin and we allow absorption in the central region only.

\section{The Computation of the Statistical Parameters}

The mean intensity for core and interface emission, respectively, is given by

$$
\begin{gathered}
\langle I\rangle=S\left(1-e^{-N q(\tau)}\right), \\
\langle I\rangle=\varepsilon \Delta s\left[\frac{2-q(\tau)}{q(\tau)}\left(1-e^{-N q(\tau)}\right)\right],
\end{gathered}
$$

where $q(\tau)=1-e^{-\tau} . S$ and $\tau$ are the source function and the optical thickness of the central layer, $\varepsilon$ and $\Delta s$ are the line emissivity and the geometrical thickness of each interface, and $N$ is the Poisson mean of the line-of-sight number of structures. The RelMSD has the same expression in both cases and is given by

$$
\delta=e^{-2 N q(\tau)} \frac{e^{N q(\tau)^{2}}-1}{\left(1-e^{-N q(\tau))^{2}} .\right.}
$$

We calculated theoretical functions $\langle I(N, \tau)\rangle$ (Figure 1) and $\delta(N, \tau)$ (Figure 2), assuming arbitrary values for the number of layers in the line of sight $(N)$ and the optical thickness of the core $(\tau)$. Concerning the RelMSD, we see 


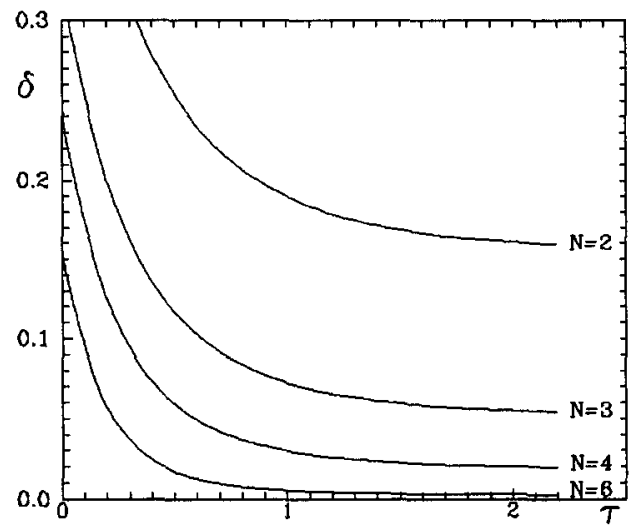

Figure 2. Theoretical relative mean square deviation, $\delta(N, \tau)$.

that it is decreasing with increasing $N$ and $\tau$. For large $\tau$ values, $\delta$ becomes insensitive to $\tau$ variations $\left(\delta=1 /\left(e^{N}-1\right)\right.$ for $\left.\tau \gg 1\right)$, and tends to 0 with growing $N$. If the central layer is optically thin $(N \tau \ll 1)$, then $\delta=1 / N$.

\section{The Statistical Parameters from Observations}

The observations we used were produced by the Harvard College Observatory spectrometer on ATM aboard Skylab. For relatively quiet regions, the observational values of the mean intensity, $\left\langle I_{\text {obs }}\right\rangle$, and RelMSD, $\delta_{\text {obs }}$, were measured. Table 1 gives $\delta_{\text {obs }}$ values for some typical prominence regions.

Table 1. Typical values for the RelMSD in the observed prominences.

\begin{tabular}{crrr} 
Spectral line & Bright regions & Normal regions & Faint regions \\
\hline Ly- $\alpha 1216$ & $0.045-0.06$ & $0.055-0.075$ & $0.075-0.09$ \\
Ly- $\beta 1026$ & $0.05-0.065$ & $0.06-0.085$ & $0.075-0.1$ \\
Ly- $\gamma 972$ & 0.07 & $0.07-0.1$ & - \\
CII 1336 & $0.1-0.25$ & $0.25-0.3$ & $0.3-0.4$ \\
OVI 1032 & $0.15-0.25$ & $0.1-0.15$ & $0.2-0.25$ \\
CIII 977 & $0.1-0.13$ & $0.065-0.1$ & 0.1 \\
OIV 554 & $0.35-0.4$ & $0.35-0.5$ & $0.5-0.6$
\end{tabular}

For the mean intensities of the studied areas, we generally find a factor of approximately 1.4 between bright and normal and between normal and faint regions, respectively.

The optically thick lines such as Ly- $\alpha$ and $\mathrm{Ly}-\beta$ exhibit smaller dispersion compared to other lines. The lowest values of $\delta_{\text {obs }}$ are found in the brightest parts of the prominences. Slightly greater values of $\delta_{\mathrm{obs}}$ are observed for the optically thin lines that are emitted by hot regions and exhibit only a small 
absorption in the core (CII $1336 \AA$, CIII $977 \AA$, OVI 1032 $\AA$ ). The RelMSD decreases as we move from the faint regions to the more intense ones, except for the brightest parts where large values occur. Higher dispersion occurs for the lines with wavelengths shorter than the Lyman limit, for instance the OIV $554 \AA$ line where the absorption in the core is high.

\section{Number of Structures on the Line of Sight}

In the optically thick limit $\delta$ does not depend on $\tau$ and enables us to estimate, from the observed values, the expected number of inhomogeneities along the line of sight. For the Ly- $\alpha$ and Ly- $\beta$ lines, $\delta=0.05-0.09$ provides $N$ from 3.2 to 2.5. The relative fluctuations in the Ly- $\beta$ line, and mostly in the Ly- $\gamma$ line $(\delta \cong 0.1)$, are slightly greater than those for the Ly- $\alpha$ line, which implies that assuming the same number of structural elements - a smaller optical thickness in these lines is required to fit the observational value of $\delta$.

For optically thin lines (CII $1336 \AA$, CIII $977 \AA$, OVI $1032 \AA$ ), the central absorbing part of the structures becomes transparent $(\tau \rightarrow 0)$ and the interface emission model gives $\delta \cong 1 / N$. A value of the RelMSD of $0.1-0.25$ implies $N=4-10$. However, for the OVI and CIII lines, the observational values of $\delta$ in the brightest regions are higher than expected. This could be due to the presence of another source of fluctuations beside the supposed one. The relatively large differences between the observational values of $\delta$ for different lines could be explained by: a) $\delta \cong 1 / N$ does not hold, or b) the possible existence of a different number of independent structural elements with various temperatures. A number of structures in the same range is also obtained from the observed distributions of radiation intensity (Pojoga et al. 1998).

\section{Conclusions}

The above results allow us to assume the existence of a small number of structures in the line of sight. More precisely, for the same prominence region a number of approximately three cool absorbing cores and 4-10 emitting envelopes are found, when the optically thick and thin lines, respectively, are involved. The difference may be due to the existence of more complicated structures than the modeled ones.

Acknowledgments. This research was supported by the French Ministry of Foreign Affairs (S.P.) and by NATO under grant CRG 960346 (A.G.N.).

\section{References}

Engvold, O., Jensen, E., Zhang, Y. and Brynildsen, N. 1989, Hvar Obs. Bull., 13(1), 205

Nikoghossian, A. G., Pojoga, S. and Mouradian, Z. 1997, A\&A, 325, 813

Orrall, F. Q. and Schmahl, E. J. 1980, ApJ, 240, 908

Pojoga, S., Nikoghossian, A. G. and Mouradian, Z. 1998, A\&A, to be submitted

Zirker, J. B. and Koutchmy, S. 1990, Sol. Phys., 127, 109 